\title{
Fibonacci Elliptic Biquaternions ${ }^{1}$
}

\author{
Kahraman Esen Özen ${ }^{1 *}$ and Murat Tosun ${ }^{2}$ \\ ${ }^{1}$ Sakarya, Turkey \\ ${ }^{2}$ Department of Mathematics, Faculty of Science and Arts, Sakarya University, Sakarya, Turkey \\ *Corresponding author
}

\section{Article Info}

Keywords: Elliptic biquaternions, $\mathrm{Fi}$ bonacci numbers, Matrix representations, Quaternions

2010 AMS: 11B39, $11 R 52$

Received: 15 October 2020

Accepted: 6 January 2021

Available online: 29 January 2021

\begin{abstract}
A. F. Horadam defined the complex Fibonacci numbers and Fibonacci quaternions in the middle of the 20th century. Half a century later, S. Halıc introduced the complex Fibonacci quaternions by inspiring from these definitions and discussed some properties of them. Recently, the elliptic biquaternions, which are generalized form of the complex and real quaternions, have been presented. In this study, we introduce the set of Fibonacci elliptic biquaternions that includes the set of complex Fibonacci quaternions as a special case, and investigate some properties of Fibonacci elliptic biquaternions. Furthermore, we give the Binet formula and Cassini's identity in terms of Fibonacci elliptic biquaternions. Finally, we give elliptic and real matrix representations of Fibonacci elliptic biquaternions.
\end{abstract}

\section{Introduction}

Real quaternions and complex quaternions were introduced by Hamilton in 1843 [1] and 1853 [2], respectively. The set of real quaternions and the set of complex quaternions are represented as

$$
H=\left\{w=w_{0}+w_{1} \mathbf{i}+w_{2} \mathbf{j}+w_{3} \mathbf{k}: w_{0}, w_{1}, w_{2}, w_{3} \in \mathbb{R}\right\}
$$

and

$$
H_{\mathbb{C}}=\left\{W=W_{0}+W_{1} \mathbf{i}+W_{2} \mathbf{j}+W_{3} \mathbf{k}: W_{0}, W_{1}, W_{2}, W_{3} \in \mathbb{C}\right\}
$$

respectively where the quaternionic units $\mathbf{i}, \mathbf{j}$ and $\mathbf{k}$ satisfy

$$
\mathbf{i}^{2}=\mathbf{j}^{2}=\mathbf{k}^{2}=-1, \mathbf{i j}=-\mathbf{j i}=\mathbf{k}, \mathbf{j k}=-\mathbf{k} \mathbf{j}=\mathbf{i}, \mathbf{k i}=-\mathbf{i k}=\mathbf{j} .
$$

As a consequence of the representations given above, a complex quaternion $W$ can be written as

$$
W=w+i w^{*}, \quad i^{2}=-1
$$

where $w$ and $w^{*}$ are real quaternions.

\footnotetext{
${ }^{1}$ This article is the completed version of the paper titled "Fibonacci Elliptic Biquaternions", which was presented as an virtual presentation at the 12th International Conference on Clifford Algebras and Their Applications in Mathematical Physics. The conference was held as an online conference between 3-7 August 2020.
}

Email addresses and ORCID numbers: kahraman.ozen1@ogr.sakarya.edu.tr, https://orcid.org/0000-0002-3299-6709 (K. E. Özen), tosun@sakarya.edu.tr, https://orcid.org/0000-0002-4888-1412 (M. Tosun) 
In 1963, $n-t h$ Fibonacci quaternion

$$
W_{n}=F_{n}+F_{n+1} \mathbf{i}+F_{n+2} \mathbf{j}+F_{n+3} \mathbf{k}
$$

was introduced by changing the components in equation (1.1) with the consecutive Fibonacci numbers by Horadam. Also, Horadam similarly defined the $n-t h$ Lucas quaternion

$$
T_{n}=L_{n}+L_{n+1} \mathbf{i}+L_{n+2} \mathbf{j}+L_{n+3} \mathbf{k} .
$$

Here $L_{n}$ and $F_{n}$ are $n-t h$ Lucas number and $n-t h$ Fibonacci number, respectively [3].

On the other hand, the complex Fibonacci numbers were defined as

$$
C_{n}=F_{n}+i F_{n+1}, \quad i^{2}=-1
$$

in [3]. Then, Halıc expanded this definition to the complex quaternions with the name " $n-t h$ complex Fibonacci quaternion". Halıc gave this definition by changing the real quaternions $w$ and $w^{*}$ in the equation (1.2) with consecutive Fibonacci quaternions $W_{n}$ and $W_{n+1}$ as in the following [4]:

$$
R_{n}=W_{n}+i W_{n+1}, \quad i^{2}=-1 .
$$

There are many studies on Fibonacci quaternions and complex Fibonacci quaternions in the literature. The readers are referred to the studies [3]-[8] for these topics.

Recently, Özen and Tosun have expressed elliptic biquaternions comprising the complex and real quaternions [9]. The set of them is given as follows:

$$
H \mathbb{C}_{p}=\left\{U=U_{0}+U_{1} \mathbf{i}+U_{2} \mathbf{j}+U_{3} \mathbf{k}: U_{0}, U_{1}, U_{2}, U_{3} \in \mathbb{C}_{p}\right\}
$$

where $\mathbb{C}_{p}=\left\{u+I v: u, v \in \mathbb{R}, I^{2}=p, p \in \mathbb{R}^{-}\right\}$indicates the set of elliptic numbers. The system of elliptic numbers is a one-parameter family of generalized complex number systems. The readers are referred to [10]-[14] for some interesting studies on the generalized complex numbers and elliptic numbers.

For any two elliptic biquaternions $U=U_{0}+U_{1} \mathbf{i}+U_{2} \mathbf{j}+U_{3} \mathbf{k} \in H \mathbb{C}_{p}$ and $V=V_{0}+V_{1} \mathbf{i}+V_{2} \mathbf{j}+V_{3} \mathbf{k} \in H \mathbb{C}_{p}$, addition and scalar multiplication by $\lambda \in \mathbb{C}_{p}$ are given by

$$
\begin{aligned}
U+V & =\left(U_{0}+V_{0}\right)+\left(U_{1}+V_{1}\right) \mathbf{i}+\left(U_{2}+V_{2}\right) \mathbf{j}+\left(U_{3}+V_{3}\right) \mathbf{k} \\
\lambda U & =\left(\lambda U_{0}\right)+\left(\lambda U_{1}\right) \mathbf{i}+\left(\lambda U_{2}\right) \mathbf{j}+\left(\lambda U_{3}\right) \mathbf{k}
\end{aligned}
$$

and also, the multiplication of $U$ and $V$ is defined as in the following [9]:

$$
\begin{aligned}
U V= & {\left[\left(U_{0} V_{0}\right)-\left(U_{1} V_{1}\right)-\left(U_{2} V_{2}\right)-\left(U_{3} V_{3}\right)\right]+\left[\left(U_{0} V_{1}\right)+\left(U_{1} V_{0}\right)+\left(U_{2} V_{3}\right)-\left(U_{3} V_{2}\right)\right] \mathbf{i} } \\
& +\left[\left(U_{0} V_{2}\right)-\left(U_{1} V_{3}\right)+\left(U_{2} V_{0}\right)+\left(U_{3} V_{1}\right)\right] \mathbf{j}+\left[\left(U_{0} V_{3}\right)+\left(U_{1} V_{2}\right)-\left(U_{2} V_{1}\right)+\left(U_{3} V_{0}\right)\right] \mathbf{k} .
\end{aligned}
$$

Moreover, the Hamiltonian, complex and total conjugates of $U$ are as below:

$$
\begin{aligned}
\bar{U} & =U_{0}-U_{1} \mathbf{i}-U_{2} \mathbf{j}-U_{3} \mathbf{k} \\
U^{*} & =U_{0}{ }^{*}+U_{1}{ }^{*} \mathbf{i}+U_{2}{ }^{*} \mathbf{j}+U_{3}{ }^{*} \mathbf{k} \\
U^{\dagger} & =U_{0}{ }^{*}-U_{1}{ }^{*} \mathbf{i}-U_{2}{ }^{*} \mathbf{j}-U_{3}{ }^{*} \mathbf{k}
\end{aligned}
$$

where superscript stars on $U_{0}, U_{1}, U_{2}$ and $U_{3}$ denote the usual complex conjugation. On the other hand, the semi-norm of $U$ is defined as [9]:

$$
N(U)=U_{0}^{2}+U_{1}^{2}+U_{2}^{2}+U_{3}^{2}
$$

In the next section, we introduce Fibonacci elliptic biquaternions and give their some properties. In the last section, we give elliptic and real matrix representations of Fibonacci elliptic biquaternions.

\section{Fibonacci elliptic biquaternions and their some properties}

Thanks to [15], we know that $p$-complex Fibonacci numbers are given as in the following:

$$
\left(C_{p}\right)_{n}=F_{n}+I F_{n+1}, I^{2}=p \in \mathbb{R}
$$


where $F_{n}$ is the $n-t h$ Fibonacci number. Here, we consider the case $p \in \mathbb{R}^{-}$and call these numbers Fibonacci elliptic numbers. Thus, $n-t h$ Fibonacci elliptic number is defined as

$$
\left(C_{p}\right)_{n}=F_{n}+I F_{n+1}, I^{2}=p \in \mathbb{R}^{-} .
$$

By following the method given in [4], we expand this definition to the elliptic biquaternions with the name " $n-t h$ Fibonacci elliptic biquaternion". That is, $n-t h$ Fibonacci elliptic biquaternion is given by

$$
\left(U_{p}\right)_{n}=W_{n}+I W_{n+1}, \quad I^{2}=p \in \mathbb{R}^{-}
$$

where

$$
W_{n}=F_{n}+F_{n+1} \mathbf{i}+F_{n+2} \mathbf{j}+F_{n+3} \mathbf{k}
$$

and

$$
W_{n+1}=F_{n+1}+F_{n+2} \mathbf{i}+F_{n+3} \mathbf{j}+F_{n+4} \mathbf{k}
$$

are consecutive Fibonacci quaternions. Therefore, $\left(U_{p}\right)_{n}$ can be written as follows:

$$
\left(U_{p}\right)_{n}=\left(F_{n}+I F_{n+1}\right)+\left(F_{n+1}+I F_{n+2}\right) \mathbf{i}+\left(F_{n+2}+I F_{n+3}\right) \mathbf{j}+\left(F_{n+3}+I F_{n+4}\right) \mathbf{k}, \quad I^{2}=p \in \mathbb{R}^{-} .
$$

As a consequence of the definition of Fibonacci elliptic numbers and the equation $(2.1),\left(U_{p}\right)_{n}$ can also be given in the following form

$$
\left(U_{p}\right)_{n}=\left(C_{p}\right)_{n}+\left(C_{p}\right)_{n+1} \mathbf{i}+\left(C_{p}\right)_{n+2} \mathbf{j}+\left(C_{p}\right)_{n+3} \mathbf{k}, \quad I^{2}=p \in \mathbb{R}^{-} .
$$

Thus, $\left(U_{p}\right)_{n}$ includes a scalar part

$$
S\left(\left(U_{p}\right)_{n}\right)=\left(C_{p}\right)_{n}
$$

and a vectorial part

$$
V\left(\left(U_{p}\right)_{n}\right)=\left(C_{p}\right)_{n+1} \mathbf{i}+\left(C_{p}\right)_{n+2} \mathbf{j}+\left(C_{p}\right)_{n+3} \mathbf{k}
$$

The Hamiltonian, complex and total conjugates of $\left(U_{p}\right)_{n}$ can be found as

$$
\begin{aligned}
& \overline{\left(U_{p}\right)_{n}}=\left(C_{p}\right)_{n}-\left(C_{p}\right)_{n+1} \mathbf{i}-\left(C_{p}\right)_{n+2} \mathbf{j}-\left(C_{p}\right)_{n+3} \mathbf{k} \\
& \left(U_{p}\right)_{n}^{*}=\left(C_{p}\right)_{n}^{*}+\left(C_{p}\right)_{n+1}^{*} \mathbf{i}+\left(C_{p}\right)_{n+2}^{*} \mathbf{j}+\left(C_{p}\right)_{n+3}^{*} \mathbf{k} \\
& \left(U_{p}\right)_{n}^{\dagger}=\left(C_{p}\right)_{n}^{*}-\left(C_{p}\right)_{n+1}^{*} \mathbf{i}-\left(C_{p}\right)_{n+2}^{*} \mathbf{j}-\left(C_{p}\right)_{n+3}^{*} \mathbf{k}
\end{aligned}
$$

where $\left(C_{p}\right)_{n}^{*}=F_{n}-I F_{n+1}$. From here, it can be easily seen that the following identities hold:

$$
\begin{aligned}
& \left(U_{p}\right)_{n}+\overline{\left(U_{p}\right)_{n}}=2\left(C_{p}\right)_{n} \\
& \left(U_{p}\right)_{n}+\left(U_{p}\right)_{n}^{*}=2 W_{n} .
\end{aligned}
$$

The semi norm of $\left(U_{p}\right)_{n}$ can be given as in the following:

$$
N\left(\left(U_{p}\right)_{n}\right)=\left(C_{p}\right)_{n}^{2}+\left(C_{p}\right)_{n+1}^{2}+\left(C_{p}\right)_{n+2}^{2}+\left(C_{p}\right)_{n+3}^{2} .
$$

If we use the identities

$$
F_{n-1}+F_{n+1}=L_{n}, \quad n \in \mathbb{Z}^{+}
$$

and

$$
F_{n}^{2}+F_{n+1}^{2}=F_{2 n+1}, n \in \mathbb{Z}
$$

given in [16], we get

$$
N\left(\left(U_{p}\right)_{n}\right)=\left(F_{2 n+1}+p F_{2 n+3}+F_{2 n+5}+p F_{2 n+7}\right)+2 I\left(F_{n+1} L_{n+1}+F_{n+3} L_{n+3}\right) .
$$

Note that we will show the set of Fibonacci elliptic biquaternions with $F H \mathbb{C}_{p}$ throughout the paper. For negative indices, the Fibonacci elliptic biquaternions can be given as in the following lemma. 
Lemma 2.1. For $\left(U_{p}\right)_{n} \in F H \mathbb{C}_{p}$, the equality

$$
\left(U_{p}\right)_{-n}=(-1)^{n}\left[\left(-F_{n}+I F_{n-1}\right)+\left(F_{n-1}-I F_{n-2}\right) \mathbf{i}+\left(-F_{n-2}+I F_{n-3}\right) \mathbf{j}+\left(F_{n-3}-I F_{n-4}\right) \mathbf{k}\right]
$$

is satisfied.

Proof. By considering the definition of the Fibonacci elliptic biquaternions and the equality $F_{-n}=(-1)^{n+1} F_{n}$ given in [17], we can write the followings:

$$
\begin{aligned}
\left(U_{p}\right)_{-n}= & \left(F_{-n}+I F_{-n+1}\right)+\left(F_{-n+1}+I F_{-n+2}\right) \mathbf{i}+\left(F_{-n+2}+I F_{-n+3}\right) \mathbf{j}+\left(F_{-n+3}+I F_{-n+4}\right) \mathbf{k} \\
= & \left(F_{-n}+I F_{-(n-1)}\right)+\left(F_{-(n-1)}+I F_{-(n-2)}\right) \mathbf{i}+\left(F_{-(n-2)}+I F_{-(n-3)}\right) \mathbf{j}+\left(F_{-(n-3)}+I F_{-(n-4)}\right) \mathbf{k} \\
= & \left((-1)^{n+1} F_{n}+I(-1)^{n} F_{n-1}\right)+\left((-1)^{n} F_{n-1}+I(-1)^{n-1} F_{n-2}\right) \mathbf{i} \\
& +\left((-1)^{n-1} F_{n-2}+I(-1)^{n-2} F_{n-3}\right) \mathbf{j}+\left((-1)^{n-2} F_{n-3}+I(-1)^{n-3} F_{n-4}\right) \mathbf{k} \\
= & (-1)^{n}\left[\left(-F_{n}+I F_{n-1}\right)+\left(F_{n-1}-I F_{n-2}\right) \mathbf{i}+\left(-F_{n-2}+I F_{n-3}\right) \mathbf{j}+\left(F_{n-3}-I F_{n-4}\right) \mathbf{k}\right]
\end{aligned}
$$

Now, we give the following theorem which reveals an essential relation between the Fibonacci numbers and Fibonacci elliptic biquaternions.

Theorem 2.2. (Binet Formula) For $\left(U_{p}\right)_{n} \in F H \mathbb{C}_{p}$, Binet formula is given as

$$
\left(U_{p}\right)_{n}=\left(U_{p}\right)_{1} F_{n}+\left(U_{p}\right)_{0} F_{n-1}
$$

where $n \geq 0$.

Proof. By direct calculation, the followings can be written easily:

$$
\begin{aligned}
\left(U_{p}\right)_{1} F_{n}+\left(U_{p}\right)_{0} F_{n-1}= & {\left[\left(F_{1}+I F_{2}\right)+\left(F_{2}+I F_{3}\right) \mathbf{i}+\left(F_{3}+I F_{4}\right) \mathbf{j}+\left(F_{4}+I F_{5}\right) \mathbf{k}\right] F_{n} } \\
& +\left[\left(F_{0}+I F_{1}\right)+\left(F_{1}+I F_{2}\right) \mathbf{i}+\left(F_{2}+I F_{3}\right) \mathbf{j}+\left(F_{3}+I F_{4}\right) \mathbf{k}\right] F_{n-1} \\
= & F_{1} F_{n}+F_{2} F_{n} \mathbf{i}+F_{3} F_{n} \mathbf{j}+F_{4} F_{n} \mathbf{k}+I\left(F_{2} F_{n}+F_{3} F_{n} \mathbf{i}+F_{4} F_{n} \mathbf{j}+F_{5} F_{n} \mathbf{k}\right) \\
& +F_{0} F_{n-1}+F_{1} F_{n-1} \mathbf{i}+F_{2} F_{n-1} \mathbf{j}+F_{3} F_{n-1} \mathbf{k}+I\left(F_{1} F_{n-1}+F_{2} F_{n-1} \mathbf{i}+F_{3} F_{n-1} \mathbf{j}+F_{4} F_{n-1} \mathbf{k}\right) \\
= & {\left[\left(F_{0} F_{n-1}+F_{1} F_{n}\right)+I\left(F_{1} F_{n-1}+F_{2} F_{n}\right)\right]+\left[\left(F_{1} F_{n-1}+F_{2} F_{n}\right)+I\left(F_{2} F_{n-1}+F_{3} F_{n}\right)\right] \mathbf{i} } \\
& +\left[\left(F_{2} F_{n-1}+F_{3} F_{n}\right)+I\left(F_{3} F_{n-1}+F_{4} F_{n}\right)\right] \mathbf{j}+\left[\left(F_{3} F_{n-1}+F_{4} F_{n}\right)+I\left(F_{4} F_{n-1}+F_{5} F_{n}\right)\right] \mathbf{k}
\end{aligned}
$$

Using the identity

$$
F_{n} F_{m}+F_{n+1} F_{m+1}=F_{m+n+1}, \quad m, n \in \mathbb{Z}^{+}
$$

given in [5] and the equalities

$$
F_{0} F_{n-1}+F_{1} F_{n}=0 F_{n-1}+1 F_{n}=F_{n}
$$

and

$$
F_{1} F_{n-1}+F_{2} F_{n}=1 F_{n-1}+1 F_{n}=F_{n-1}+F_{n}=F_{n+1},
$$

the proof is completed.

Theorem 2.3. (Cassini's Identity) For $\left(U_{p}\right)_{n} \in F H \mathbb{C}_{p}$, Cassini's identity is given as

$$
\left(U_{p}\right)_{n-1}\left(U_{p}\right)_{n+1}-\left(U_{p}\right)_{n}^{2}=(-1)^{n}\left(2 W_{1}-3 \mathbf{k}-p F_{1}^{2} T_{0}-p F_{2} W_{0}+3 p F_{2}\right)+I\left(W_{n-1} W_{n+2}-W_{n} W_{n+1}\right)
$$

where $n \geq 1$.

Proof. By direct calculation, we get

$$
\begin{aligned}
\left(U_{p}\right)_{n-1}\left(U_{p}\right)_{n+1}-\left(U_{p}\right)_{n}^{2} & =\left(W_{n-1}+I W_{n}\right)\left(W_{n+1}+I W_{n+2}\right)-\left(W_{n}+I W_{n+1}\right)^{2} \\
& =W_{n-1} W_{n+1}+p W_{n} W_{n+2}+I\left(W_{n} W_{n+1}+W_{n-1} W_{n+2}\right)-\left(W_{n}^{2}+p W_{n+1}^{2}+2 I W_{n} W_{n+1}\right) \\
& =W_{n-1} W_{n+1}-W_{n}^{2}+p\left(W_{n} W_{n+2}-W_{n+1}^{2}\right)+I\left(W_{n-1} W_{n+2}-W_{n} W_{n+1}\right) .
\end{aligned}
$$


Using the identities

$$
W_{n-1} W_{n+1}-W_{n}^{2}=(-1)^{n}\left(2 W_{1}-3 \mathbf{k}\right)
$$

and

$$
W_{n+1-r} W_{n+1+r}-W_{n+1}^{2}=(-1)^{n-r}\left[F_{r}^{2} T_{0}+F_{2 r}\left(W_{0}-3 r\right)\right] \quad(\text { for } r=1)
$$

given in [5], the proof is completed.

\section{Matrix representations of Fibonacci elliptic biquaternions}

In this section, elliptic and real matrix representations of Fibonacci elliptic biquaternions are given by emphasizing the isomorphisms which determine these matrix representations.

\section{1. $2 \times 2$ and $4 \times 4$ elliptic matrix representations of Fibonacci elliptic biquaternions}

Thanks to Özen and Tosun [18], we know that there is a faithful relation between the elliptic biquaternions and 2x2 elliptic matrices. Every elliptic biquaternion $U=U_{0}+U_{1} \mathbf{i}+U_{2} \mathbf{j}+U_{3} \mathbf{k} \in H \mathbb{C}_{p}$ has a $2 \times 2$ elliptic matrix representation

$$
\sigma(U)=\left[\begin{array}{cc}
U_{0}+\frac{1}{\sqrt{|p|}} I U_{1} & -U_{2}-\frac{1}{\sqrt{|p|}} I U_{3} \\
U_{2}-\frac{1}{\sqrt{|p|}} I U_{3} & U_{0}-\frac{1}{\sqrt{|p|}} I U_{1}
\end{array}\right]
$$

which is determined by means of the following linear isomorphism [18]

$$
\begin{aligned}
\sigma: H \mathbb{C}_{p} & \rightarrow M_{2 \times 2}\left(\mathbb{C}_{p}\right) \\
U & \rightarrow \sigma(U)=\left[\begin{array}{ll}
U_{0}+\frac{1}{\sqrt{|p|}} I U_{1} & -U_{2}-\frac{1}{\sqrt{|p|}} I U_{3} \\
U_{2}-\frac{1}{\sqrt{|p|}} I U_{3} & U_{0}-\frac{1}{\sqrt{|p|}} I U_{1}
\end{array}\right] .
\end{aligned}
$$

Let us consider the restriction of this isomorphism to the set of Fibonacci elliptic biquaternions. Then we get the following isomorphism:

$$
\begin{aligned}
\sigma^{*}: F H \mathbb{C}_{p} \rightarrow \sigma\left(F H \mathbb{C}_{p}\right) & \subset M_{2 \times 2}\left(\mathbb{C}_{p}\right) \\
\left(U_{p}\right)_{n} \rightarrow \sigma^{*}\left(\left(U_{p}\right)_{n}\right) & =\left[\begin{array}{cc}
\left(C_{p}\right)_{n}+\frac{1}{\sqrt{|p|}} I\left(C_{p}\right)_{n+1} & -\left(C_{p}\right)_{n+2}-\frac{1}{\sqrt{|p|}} I\left(C_{p}\right)_{n+3} \\
\left(C_{p}\right)_{n+2}-\frac{1}{\sqrt{|p|}} I\left(C_{p}\right)_{n+3} & \left(C_{p}\right)_{n}-\frac{1}{\sqrt{|p|}} I\left(C_{p}\right)_{n+1}
\end{array}\right] .
\end{aligned}
$$

Thus, we can give the following definition by using the equality $I^{2}=p=-\sqrt{|p|} \sqrt{|p|}$.

Definition 3.1. The matrix

$$
\left[\begin{array}{cc}
\left(F_{n}-\sqrt{|p|} F_{n+2}\right)+I\left(1+\frac{1}{\sqrt{|p|}}\right) F_{n+1} & \left(-F_{n+2}+\sqrt{|p|} F_{n+4}\right)-I\left(1+\frac{1}{\sqrt{|p|}}\right) F_{n+3} \\
\left(F_{n+2}+\sqrt{|p|} F_{n+4}\right)+I\left(1-\frac{1}{\sqrt{|p|}}\right) F_{n+3} & \left(F_{n}+\sqrt{|p|} F_{n+2}\right)+I\left(1-\frac{1}{\sqrt{|p|}}\right) F_{n+1}
\end{array}\right]
$$

derived from $\sigma^{*}\left(\left(U_{p}\right)_{n}\right)$ is called $2 x 2$ elliptic matrix representation of $\left(U_{p}\right)_{n}$.

On the other hand, there is an isomorphism between the matrix space $M=\left\{\left[\begin{array}{cccc}X_{0} & -X_{1} & -X_{2} & -X_{3} \\ X_{1} & X_{0} & -X_{3} & X_{2} \\ X_{2} & X_{3} & X_{0} & -X_{1} \\ X_{3} & -X_{2} & X_{1} & X_{0}\end{array}\right]: X_{0}, X_{1}, X_{2}, X_{3} \in \mathbb{C}_{p}\right\}$ and the elliptic biquaternion space $H \mathbb{C}_{p}$ [19]:

$$
\begin{aligned}
& \gamma: H \mathbb{C}_{p} \rightarrow M \\
& U \rightarrow \gamma(U)=\left[\begin{array}{cccc}
U_{0} & -U_{1} & -U_{2} & -U_{3} \\
U_{1} & U_{0} & -U_{3} & U_{2} \\
U_{2} & U_{3} & U_{0} & -U_{1} \\
U_{3} & -U_{2} & U_{1} & U_{0}
\end{array}\right]
\end{aligned}
$$


Similarly above, if this isomorphism is restricted to the set of Fibonacci elliptic biquaternions, it is not difficult to see the following isomorphism

$$
\begin{aligned}
\gamma^{*}: F H \mathbb{C}_{p} & \rightarrow \gamma\left(F H \mathbb{C}_{p}\right) \subset M \\
\left(U_{p}\right)_{n} \rightarrow \gamma^{*}\left(\left(U_{p}\right)_{n}\right) & =\left[\begin{array}{cccc}
\left(C_{p}\right)_{n} & -\left(C_{p}\right)_{n+1} & -\left(C_{p}\right)_{n+2} & -\left(C_{p}\right)_{n+3} \\
\left(C_{p}\right)_{n+1} & \left(C_{p}\right)_{n} & -\left(C_{p}\right)_{n+3} & \left(C_{p}\right)_{n+2} \\
\left(C_{p}\right)_{n+2} & \left(C_{p}\right)_{n+3} & \left(C_{p}\right)_{n} & -\left(C_{p}\right)_{n+1} \\
\left(C_{p}\right)_{n+3} & -\left(C_{p}\right)_{n+2} & \left(C_{p}\right)_{n+1} & \left(C_{p}\right)_{n}
\end{array}\right] .
\end{aligned}
$$

Then, we can give the following according to definition of $n-t h$ Fibonacci elliptic number.

Definition 3.2. The matrix

$$
\left[\begin{array}{cccc}
F_{n}+I F_{n+1} & -F_{n+1}-I F_{n+2} & -F_{n+2}-I F_{n+3} & -F_{n+3}-I F_{n+4} \\
F_{n+1}+I F_{n+2} & F_{n}+I F_{n+1} & -F_{n+3}-I F_{n+4} & F_{n+2}+I F_{n+3} \\
F_{n+2}+I F_{n+3} & F_{n+3}+I F_{n+4} & F_{n}+I F_{n+1} & -F_{n+1}-I F_{n+2} \\
F_{n+3}+I F_{n+4} & -F_{n+2}-I F_{n+3} & F_{n+1}+I F_{n+2} & F_{n}+I F_{n+1}
\end{array}\right]
$$

derived from $\gamma^{*}\left(\left(U_{p}\right)_{n}\right)$ is called $4 x 4$ elliptic matrix representation of $\left(U_{p}\right)_{n}$.

\subsection{8x8 real matrix representations of Fibonacci elliptic biquaternions}

In the study [20], Özen and Tosun obtained 8x8 real matrix representation of an arbitrary elliptic biquaternion in the space $H \mathbb{C}_{p}$. However, the isomorphism that determines this representation was not emphasized with its domain and range in this study. So, we are not able to apply the method of restriction as in the previous subsection. Here we give particular importance to get an isomorphism whose domain is $F H \mathbb{C}_{p}$ and whose range is a special 8x8 real matrix set. Because of this, we need some preparation.

From [21], it is known that there is an isomorphism between the elliptic matrix set $M_{4 \times 4}\left(\mathbb{C}_{p}\right)$ and real matrix set $M_{8 \times 8}^{\Omega}(\mathbb{R})$ as in the following:

$$
\begin{aligned}
& \psi: M_{4 \times 4}\left(\mathbb{C}_{p}\right) \rightarrow M_{8 \times 8}^{\Omega}(\mathbb{R}) \\
& A=A_{1}+I A_{2} \rightarrow \psi(A)=\left[\begin{array}{cc}
A_{1} & -\sqrt{|p|} A_{2} \\
\sqrt{|p|} A_{2} & A_{1}
\end{array}\right]
\end{aligned}
$$

where $M_{8 \times 8}^{\Omega}(\mathbb{R})=\left\{\left[\begin{array}{cc}G & -\sqrt{|p|} H \\ \sqrt{|p|} H & G\end{array}\right]: G, H \in M_{4 \times 4}(\mathbb{R})\right\}$.

Since $\gamma\left(F H \mathbb{C}_{p}\right) \subset M \subset M_{4 \times 4}\left(\mathbb{C}_{p}\right)$, we can restrict the isomorphism $\psi$ to the set $\gamma\left(F H \mathbb{C}_{p}\right)$. If we do this, we have the isomorphism $\psi^{*}: \gamma\left(F H \mathbb{C}_{p}\right) \rightarrow \psi\left(\gamma\left(F H \mathbb{C}_{p}\right)\right) \subset M_{8 \times 8}^{\Omega}(\mathbb{R})$. To obtain 8x8 real matrix representations of Fibonacci elliptic biquaternions is the aim of us. To do so, we write $\gamma^{*}\left(\left(U_{p}\right)_{n}\right)$ as follows:

$$
\gamma^{*}\left(\left(U_{p}\right)_{n}\right)=B_{1}(n)+I B_{2}(n)
$$

where

$$
B_{1}(n)=\left[\begin{array}{cccc}
F_{n} & -F_{n+1} & -F_{n+2} & -F_{n+3} \\
F_{n+1} & F_{n} & -F_{n+3} & F_{n+2} \\
F_{n+2} & F_{n+3} & F_{n} & -F_{n+1} \\
F_{n+3} & -F_{n+2} & F_{n+1} & F_{n}
\end{array}\right] \in M_{4 \times 4}(\mathbb{R})
$$

and

$$
B_{2}(n)=\left[\begin{array}{cccc}
F_{n+1} & -F_{n+2} & -F_{n+3} & -F_{n+4} \\
F_{n+2} & F_{n+1} & -F_{n+4} & F_{n+3} \\
F_{n+3} & F_{n+4} & F_{n+1} & -F_{n+2} \\
F_{n+4} & -F_{n+3} & F_{n+2} & F_{n+1}
\end{array}\right] \in M_{4 \times 4}(\mathbb{R}) .
$$

Then, the compound function $\delta=\psi^{*} \circ \gamma^{*}$ yields the following isomorphism:

$$
\begin{aligned}
\delta: F H \mathbb{C}_{p} & \rightarrow \psi\left(\gamma\left(F H \mathbb{C}_{p}\right)\right) \subset M_{8 \times 8}^{\Omega}(\mathbb{R}) \\
\left(U_{p}\right)_{n} & \rightarrow \delta\left(\left(U_{p}\right)_{n}\right)=\left[\begin{array}{cc}
B_{1}(n) & -\sqrt{|p|} B_{2}(n) \\
\sqrt{|p|} B_{2}(n) & B_{1}(n)
\end{array}\right] .
\end{aligned}
$$

Consequently, we can give the following definition. 
Definition 3.3. The real matrix

\begin{tabular}{|c|c|c|c|c|c|c|c|}
\hline$F_{n}$ & $-F_{n+1}$ & $-F_{n+2}$ & $-F_{n+3}$ & $-\sqrt{|p|} F_{n+1}$ & $\sqrt{|p|} F_{n+3}$ & $\sqrt{|p|} F_{n+3}$ & $\sqrt{|p|} F_{n+4}$ \\
\hline$F_{n+1}$ & $F_{n}$ & $-F_{n+3}$ & $F_{n+2}$ & $-\sqrt{|p| F_{n+2}}$ & $\sqrt{|p| F_{n+4}}$ & $\sqrt{|p| F_{n+4}}$ & $-\sqrt{|p|} F_{n+3}$ \\
\hline$F_{n+2}$ & $F_{n+3}$ & $F_{n}$ & $-F_{n+1}$ & $-\sqrt{|p|} F_{n+3}$ & $-\sqrt{|p|} F_{n+1}$ & $-\sqrt{|p|} F_{n+1}$ & $\sqrt{|p|} F_{n+2}$ \\
\hline$F_{n+3}$ & $-F_{n+2}$ & $F_{n+1}$ & $F_{n}$ & $-\sqrt{|p|} F_{n+4}$ & $-\sqrt{|p|} F_{n+2}$ & $-\sqrt{|p|} F_{n+2}$ & $-\sqrt{|p|} F_{n+1}$ \\
\hline$\sqrt{|p|} F_{n+1}$ & $-\sqrt{|p|} F_{n+2}$ & $-\sqrt{|p|} F_{n+3}$ & $-\sqrt{|p|} F_{n+4}$ & $F_{n}$ & $-F_{n+1}$ & $-F_{n+2}$ & $-F_{n+3}$ \\
\hline$\sqrt{|p|} F_{n+2}$ & $\sqrt{|p|} F_{n+1}$ & $-\sqrt{|p|} F_{n+4}$ & $\sqrt{|p|} F_{n+3}$ & $F_{n+1}$ & $F_{n}$ & $-F_{n+3}$ & $F_{n+2}$ \\
\hline$\sqrt{|p|} F_{n+3}$ & $\sqrt{|p|} F_{n+4}$ & $\sqrt{|p|} F_{n+1}$ & $-\sqrt{|p|} F_{n+2}$ & $F_{n+2}$ & $F_{n+3}$ & $F_{n}$ & $-F_{n+1}$ \\
\hline$-\sqrt{|p|} F_{n+4}$ & $-\sqrt{|p|} F_{n+3}$ & $\sqrt{|p|} F_{n+2}$ & $\sqrt{|p|} F_{n+1}$ & $F_{n+3}$ & $-F_{n+2}$ & $F_{n+1}$ & $F_{n}$ \\
\hline
\end{tabular}

derived from $\delta\left(\left(U_{p}\right)_{n}\right)$ is called $8 x 8$ real matrix representation of $\left(U_{p}\right)_{n}$.

\section{Conclusion}

In this study, Fibonacci elliptic biquaternions and their some properties are introduced. Also, Binet formula and Cassini's identity are given in terms of Fibonacci elliptic biquaternions. Moreover, real and elliptic matrix representations of Fibonacci elliptic biquaternions are derived.

When $p=-1$, the set of elliptic numbers matches up with the set of complex numbers. In that case, the set of elliptic biquaternions is reduced to the set of complex quaternions. Therefore, Fibonacci elliptic biquaternions can be seen as generalized form of complex Fibonacci quaternions that take an important place in the literature.

The use of matrix techniques gives us many advantages in many areas of science. In this respect, this study can be seen as the first step of the future studies which will be presented by using the matrix representations of Fibonacci elliptic biquaternions.

\section{References}

[1] B. L. van der Waerden, Hamilton's discovery of quaternions, Math. Mag., 49 (1976), 227-234.

[2] W. R. Hamilton, Lectures on quaternions, Hodges and Smith, Dublin, 1853.

[3] A. F. Horadam, Complex Fibonacci numbers and Fibonacci quaternions, Am. Math. Mon., 70 (1963), $289-291$.

[4] S. Halıcı, On complex Fibonacci quaternions, Adv. Appl. Clifford Algebras, 23 (2013), 105-112.

[5] M. R. Iyer, Some results on Fibonacci quaternions, Fibonacci Q., 7 (1969), 201-210.

[6] T. Erişir, M. A. Güngör, On Fibonacci spinors, Int. J. Geom. Methods Mod. (2020), DOI: 10.1142/S0219887820500656.

[7] M. Akyig̃it, H. H. Kösal, M. Tosun, Fibonacci generalized quaternions, Adv. Appl. Clifford Algebras, 24 (2014), 631-641.

[8] C. Flaut, V. Shpakivskyi, Real matrix representations for the complex quaternions, Adv. Appl. Clifford Algebras, 23 (2013), $657-671$.

[9] K. E. Özen, M. Tosun, Elliptic biquaternion algebra, AIP Conf. Proc., 1926 (2018), 020032.

[10] I. M. Yaglom, Complex Numbers in Geometry, Academic Press, Newyork, 1968.

[11] A. A. Harkin, J. B. Harkin, Geometry of generalized complex numbers, Math. Mag., 77(2) (2004), 118-129.

[12] K. Eren, S. Ersoy, Burmester theory in Cayley-Klein planes with affine base, J. Geom., 109(3) (2018), 45.

[13] K. Eren, S. Ersoy, Revisiting Burmester theory with complex forms of Bottema's instantaneous invariants, Complex Var. Elliptic Equ., 62(4) (2017), Z. Derin, M. A. Güngör, On Lorentz transformations with elliptic biquaternions, In: Hvedri, I. (ed.) Tblisi-Mathematics, pp 121-140. Sciendo, Berlin (2020).

[15] Y. Kulaç, M. Tosun, Some equations on p-complex Fibonacci numbers, AIP Conf. Proc., 1926 (2018), 020024.

[16] R. A. Dunlap, The golden ratio and Fibonacci numbers, World Scientific, 1997.

[17] T. Koshy, Fibonacci and Lucas numbers with applications, A Wiley-Interscience Publication, U.S.A, 2001.

[18] K. E. Özen, M. Tosun, Further results for elliptic biquaternions, Conf. Proc. Sci. Technol., 1 (2018), 20-27.

[19] K. E. Özen, M. Tosun, Elliptic matrix representations of elliptic biquaternions and their applications, Int. Electron. J. Geom., 11 (2018), $96-103$.

[20] K. E. Özen, M. Tosun, A general method for solving linear elliptic biquaternion equations, Complex Var. Elliptic Equ., (2020), 1-12, DOI: $10.1080 / 17476933.2020 .1738409$.

[21] K. E. Özen, A general method for solving linear matrix equations of elliptic biquaternions with applications, AIMS Math., 5 (2020), $2211-2225$. 